Service social

\title{
Les services intensifs : une nouvelle approche dans l'intervention auprès des familles à risque
}

\section{Gaby Carrier, Marie-Christine Saint-Jacques, Laurent Chabot et Michel Thibault}

Volume 41, numéro 3, 1992

Intervenir en contexte d'autorité

URI : https://id.erudit.org/iderudit/706584ar

DOI : https://doi.org/10.7202/706584ar

Aller au sommaire du numéro

Éditeur(s)

École de service social de l'Université Laval

ISSN

1708-1734 (numérique)

Découvrir la revue

Citer cet article

Carrier, G., Saint-Jacques, M.-C., Chabot, L. \& Thibault, M. (1992). Les services intensifs : une nouvelle approche dans l'intervention auprès des familles à risque. Service social, 41(3), 41-75. https://doi.org/10.7202/706584ar

\section{Résumé de l'article}

L'augmentation du nombre de placements d'enfants et l'échec relatif de cette mesure dans le traitement des problèmes familiaux amènent les professionnels des services sociaux à réfléchir sur de nouvelles façons d'aider les familles à résoudre leurs difficultés tout en maintenant l'unité familiale intacte. Les services intensifs à la famille appartiennent à ce courant de renouveau. Le présent article décrit cette nouvelle forme d'intervention. Plus précisément, trois grands thèmes sont abordés. Le premier porte sur l'historique de ces programmes, leur philosophie et leurs principes, de même que sur les trois principaux modèles mis en oeuvre sous le vocable des services intensifs à la famille, soit les programmes Homebuilders, Familieset Family Treatment.

Dans un second temps, l'efficacité des programmes de sauvegarde de la famille est examinée en apportant une attention particulière aux problèmes méthodologiques que soulève leur évaluation. L'article se termine par une présentation des conditions nécessaires à l'implantation de ces programmes dans les services sociaux québécois. 


\section{Les services intensifs: une nouvelle approche dans l'intervention auprès des familles à risque}

Gaby CARRIER

Chercheuse

Centre de recherche sur les services communautaires

Université Laval

Marie-Christine SAINT-JACQUES Professionnelle de recherche Centre de recherche sur les services communautaires Université Laval

Laurent $\mathrm{CHABOT}$

Conseiller

Direction des services professionnels

CPEJ Richelieu

Michel THIBAULT

Conseiller

Direction des services professionnels

CPEJ de l'Estrie

\section{INTRODUCTION}

C'est à la suite du constat que trop d'enfants étaient placés en milieu substitut et que le suivi dont ils bénéficiaient était déficient que se sont développées de nouvelles stratégies visant à prévenir le placement (Pecora et al., 1990). Le présent article a pour objectif de décrire les programmes de sauvegarde 
de la famille ${ }^{1}$ actuellement en pleine expansion aux États-Unis (family preservation programs). II s'agit d'un modèle préconisant une intervention intensive, limitée dans le temps, offerte aux familles qui font face à une crise où un enfant est sur le point d'être placé dans un milieu substitut. Cette description sera complétée par une réflexion sur l'efficacité et les conditions de l'implantation d'un tel programme en milieu québécois.

Nous présenterons successivement une description des principales origines et caractéristiques de ces programmes, quelques résultats et limites des évaluations qui en ont été faites ainsi que les principales conditions d'implantation de ce type de services dans les services sociaux québécois².

\section{HISTORIQUE}

Les services de sauvegarde de la famille ne sont pas le produit spontané d'une réflexion révélatrice, mais le dernier résultat d'une lente et complexe évolution qui s'est accélérée principalement au cours des deux dernières décennies. C'est à la suite d'un examen approfondi du système de bien-être à l'enfance américain, au milieu des années 70 , que l'on en est venu à la conclusion que les enfants étaient mal servis par le système de services sociaux et que l'on ne réussissait pas à améliorer leur bien-être ; en effet, de plus en plus d'enfants étaient placés, déplacés de ressource en ressource, parfois oubliés en milieu substitut.

Soutenu par des recherches sur l'importance de la continuité parentale (Goldstein, Freud et Solnit, 1973), sur l'incapacité relative du système à assurer une permanence dans le projet de vie de l'enfant (National Commission of Children in Need of Parents, 1979) et sur certains effets négatifs du placement d'enfants (Fanshel et Shinn, 1978), un nouveau mouvement des services de bien-être s'est amorcé. La planification d'un projet de vie permanent, concept central de cette réforme, a suscité un ensemble de mesures destinées à garder les enfants dans leur foyer ou à les aider à y retourner le plus tôt possible. Parmi ces mesures, notons la loi de 1980 (Adoption Assistance and

1. L'expression « sauvegarde de la famille » est la traduction française de l'expression anglaise «family preservation ». Nous I'utilisons dans ce texte pour caractériser une approche globale issue d'une philosophie de services offerts, non pas pour maintenir l'unité familiale à tout prix, au détriment du bien-être et de la sécurité d'un enfant, mais pour amener les parents et les enfants à trouver les ressources nécessaires pour faire face à leurs problèmes familiaux ensemble.

2. Des parties de cet article sont tirées de la traduction d'un document produit à l'occasion du symposium de la Fondation de recherche et d'éducation et de l'Association américaine de thérapie familiale et conjugale (AAMFT) intitulé Family Impact Seminar et préparé par T. Ooms, D. Beck et L. Herendeen. Ce document, traduit en 1991 par M. Laurent Chabot, est intitulé Garder ensemble les familles en difficulté : programmes prometteurs et réformes d'États. 
Child Welfare $A c t$ ), le développement des services à domicile centrés sur la famille, la réallocation des ressources humaines et financières et un nouveau partage fiscal entre les divers niveaux de gouvernement (Ooms et al., 1990).

\section{Les origines des programmes de sauvegarde de la famille}

Les programmes de sauvegarde de la famille ont puisé une grande partie de leurs attributs et principes à trois importants mouvements de l'histoire des services sociaux : la planification d'un projet de vie permanent (permanency planning) pour les enfants, les services à domicile centrés sur la famille et la thérapie familiale. Nous illustrons brièvement ci-après comment ces mouvements ont contribué à l'émergence de ce type de services.

\section{La planification d'un projet de vie permanent pour l'enfant}

La « planification d'un projet de vie permanent » repose sur un ensemble de mesures et d'interventions qui amènent les intervenants à « agir de façon précoce, décisive, orientée vers l'action, afin de maintenir les enfants dans leurs foyers ou de les placer de façon permanente dans des familles d'accueil » (Maluccio et al., 1986). L'efficacité de ces mesures a été démontrée de façon éloquente dans deux projets pilotes, en Oregon et à Alameda en Californie, où les travailleurs sociaux, responsables de charges de cas allégées, ont donné aux familles des services intensifs, d'orientation behaviorale, afin d'améliorer leurs attitudes et comportements parentaux. Dans les cas d'incapacité parentale ou de désengagement important, ces intervenants déployaient aussi plus d'efforts pour mettre fin aux droits parentaux. Ces deux projets, qui ont obtenu d'intéressants résultats, étaient alors destinés à des familles dont les enfants étaient déjà placés. Par la suite, le principe de la planification d'un projet de vie permanent a évolué et plusieurs de ses mesures furent modifiées et réorientées progressivement vers un objectif de prévention du premier placement. Ce virage « préventif » a reçu de la fondation Edna McConnell Clark le nom de Family Preservation, en 1985. On reconnaissait ainsi que les efforts des services sociaux devaient viser en priorité la sauvegarde du milieu familial.

\section{Les services à domicile centrés sur la famille}

Le second apport aux programmes de sauvegarde de la famille vient des services à domicile ou encore des services centrés sur la famille, tels qu'ils ont été développés au cours des années 50 et 60 . L'illustration la plus connue de ce type de services est le Family-Centered Project, de St. Paul, Minnesota. Les professionnels qui participaient à ce programme étaient spécialement formés pour intervenir auprès des familles défavorisées présentant des 
problèmes multiples. Ils offraient des services intensifs, non traditionnels dans les foyers des familles. Ce type de services visait principalement à faire échec à l'impuissance apprise et très enracinée chez les clients et à créer une relation moins paternaliste et empreinte de dépendance envers les travailleurs sociaux. Comme ces intervenants travaillaient auprès de familles où les enfants risquaient davantage d'être placés, on commença vite à percevoir cette forme $d^{\prime}$ intervention comme une réponse logique à la planification d'un projet de vie permanent pour l'enfant et, par la suite, on fit le lien avec les services offerts dans les programmes de sauvegarde de la famille (Ooms et al., 1990 ; traduit par Chabot, 1991).

\section{L'influence du mouvement de la thérapie familiale}

Le troisième apport aux programmes de sauvegarde de la famille vient du mouvement de la thérapie familiale, qui s'est développé à la fin des années 60 et au cours des années 70, et qui a permis de mettre en évidence que les problèmes des personnes pouvaient être mieux compris et résolus dans le contexte de leurs relations intimes, particulièrement dans leur famille. Trois domaines de recherche et de pratique ont appuyé le développement des connaissances dans ce domaine : celui des relations familiales, celui du comportement et du fonctionnement des enfants ainsi que celui des désordres mentaux chroniques, particulièrement de la schizophrénie. Le mouvement de la thérapie familiale nous a ainsi appris que la meilleure façon d'aider un enfant était de renforcer sa famille.

Les thérapeutes familiaux qui ont apporté le plus aux programmes de sauvegarde de la famille ont travaillé auprès des familles défavorisées appartenant à des minorités ethniques et auprès d'enfants ou d'adolescents ayant eu des expériences de délinquance ou de troubles du comportement. L'identification de problèmes familiaux et de difficultés d'apprentissage chez ces enfants a amené ces professionnels à intégrer, dans leurs interventions, la théorie de l'apprentissage social (théorie de la socialisation) et les méthodes de traitement de l'approche systémique. Les résultats ont été impressionnants et, surtout, ont démontré que les familles défavorisées avaient des forces et pouvaient bénéficier de la thérapie. Cette confiance à redonner le pouvoir aux familles et à leur permettre de contrôler leur destinée constitue l'une des caractéristiques fondamentales des programmes de sauvegarde de la famille (Ooms et al., 1990 ; traduit par Chabot, 1991).

\section{LA PHILOSOPHIE ET LES PRINCIPES}

Les objectifs poursuivis par ces programmes sont nombreux : ils visent principalement à assurer la sécurité du jeune et de sa famille et à protéger la com- 
munauté contre le jeune, à améliorer le fonctionnement de la famille afin de prévenir le placement, à améliorer les soins offerts en milieu substitut, à faire participer davantage les parents à la prise de décision du placement, à accrô̂tre leur participation au cours du placement et à stabiliser la situation du jeune.

Par ailleurs, l'approche que préconisent les services offerts dans les programmes de sauvegarde de la famille repose sur un ensemble de principes et de postulats qui se retrouvent dans tous les programmes, peu importe qu'il s'agisse d'un modèle Homebuilders, Families ou Family Treatment. Nous les présentons ici afin de bien mettre en évidence les fondements qui doivent guider les intervenants et les administrateurs de ces programmes.

En premier lieu, les familles sont traitées avec respect, et on doit reconnaître qu'elles ont des forces et des habiletés. L'intervenant doit découvrir ce potentiel et s'appuyer sur les forces tant des membres de la famille que du milieu qui l'entoure. Ainsi, les relations que la famille entretient avec la parenté, I'appartenance culturelle et ethnique constituent autant d'éléments qui peuvent apporter un éclairage et un soutien à l'intervention. Le rôle de I'intervenant est d'agir comme catalyseur, afin de redonner aux parents le contrôle des événements et la capacité de prendre soin de leurs enfants.

En second lieu, l'évaluation et le traitement offert portent sur l'entité familiale complète, y compris la parenté, et l'enfant est considéré comme un élément intégral du système familial. De plus, la famille est vue comme un élément de sa communauté et du contexte environnemental plus large, qui comprend le milieu de travail, les ressources communautaires, bref tous les systèmes avec lesquels la famille peut interagir.

En troisième lieu, les services offerts aux familles sont généralement précis et orientés vers des buts particuliers limités, définis par la famille et I'intervenant et déterminés en fonction des besoins de la famille et non par rapport à des normes d'admissibilité à certains programmes. Cette ouverture fait naître une très grande diversité de services et d'interventions : information, référence, thérapie, soutien, counseling, services concrets.

La quatrième caractéristique des programmes de sauvegarde de la famille se rapporte aux conditions de travail. Tout d'abord, les intervenants rejoignent la famille dans son environnement, soit à la maison ou dans les autres lieux qu'elle fréquente (p. ex. : I'école, le restaurant). Il est donc plutôt exceptionnel de faire venir la famille ou l'un de ses membres au bureau pour une entrevue. De plus, les horaires de travail sont flexibles et l'intervenant doit être disponible à toute heure du jour ou de la nuit. Il y a donc place pour l'urgence. Les services peuvent être plus intensifs au début du traitement, lorsque la crise survient, et se stabiliser par la suite. L'intensité et la variété des services offerts exigent donc des charges de cas minimales, du soutien et une formation adaptée (Ooms et al., 1990 ; traduit par Chabot, 1991). 


\section{LES MODÈLES DE PROGRAMMES DE SAUVEGARDE DE LA FAMILLE}

Les services de sauvegarde de la famille ont reçu diverses appellations, selon les programmes ou les lieux où ils étaient offerts : les services de prévention de placement centrés sur la famille (family prevention services), les services intensifs de sauvegarde de la famille (intensive family preservation services), les services à domicile aux familles (home-based family services), etc. Les programmes de sauvegarde de la famille auxquels nous faisons référence dans cet article sont caractérisés par des interventions intensives dans le temps, offertes aux familles qui font face à une crise où un enfant est sur le point d'être retiré de son foyer et placé en milieu substitut. S'appuyant sur une analyse de onze programmes de cette nature, le National Resource Center on Family-Based Services, de l'Université de l'lowa, propose trois modèles de base, selon les théories ou les approches privilégiées : les programmes Homebuilders, qui offrent des services d'intervention de crise basés sur la théorie de l'apprentissage social, les programmes Families, qui offrent des services à domicile basés sur la théorie des systèmes, et les programmes Family Treatment, qui offrent des services basés sur la théorie des systèmes familiaux, mais qui se distinguent des deux autres par l'accent mis sur les interventions thérapeutiques.

Les programmes Homebuilders, lancés dans l'État de Washington, sont les plus connus et les plus répandus aux États-Unis. Ils partent du postulat que la crise conduisant à envisager le placement d'un enfant est une occasion de changement et que les parents sont plus disposés à apprendre et à changer lorsque leurs mécanismes habituels de résolution de problèmes ne fonctionnent plus. Les services offerts dans ces programmes visent à modifier les comportements parentaux et à développer des habiletés éducatives. Les objectifs du traitement sont fixés avec les parents, selon leurs priorités et leurs perceptions du problème, et on encourage les intervenants à respecter ces besoins et ces perceptions. Les services sont offerts au foyer, ils sont très intensifs, d'une durée limitée et fréquemment de nature concrète.

Les programmes Families, nés en lowa, ont été conçus pour offrir aux adolescents une solution de rechange au placement en dehors du domicile familial. On note aussi qu'ils ont été d'abord implantés en milieu rural. Basés sur la théorie des systèmes familiaux, ces programmes considèrent que la demande de placement est indicatrice de problèmes sous-jacents dans la dynamique familiale et que le retrait de l'enfant de sa famille représente le moyen choisi par les membres de la famille pour résoudre ces problèmes. Tout comme dans le cas des programmes Homebuilders, la famille participe à l'évaluation de la situation et à la définition des objectifs. Les services sont offerts dans le milieu familial, afin que les comportements soient adaptés au vécu et que la famille accroisse son pouvoir sur son environnement, mais ils sont moins intensifs que les services offerts dans les programmes Homebuilders. 
Enfin, les programmes Family Treatment, créés en Oregon, s'appuient aussi sur la théorie des systèmes familiaux et interprètent les problèmes d'une personne comme étant la manifestation d'autres problèmes familiaux. Ces programmes se distinguent des deux autres surtout par les approches thérapeutiques qu'ils préconisent. L'évaluation, entre autres, se fait généralement au moyen d'une entrevue familiale structurée. Les services sont moins intensifs que ceux des programmes Homebuilders et plus intensifs que ceux des programmes Families, et ils peuvent se donner au bureau ou au foyer (Ooms et al., 1990 ; traduit par Chabot, 1991).

Comme on peut le constater à la lecture du tableau 1 de la page suivante, les différences entre ces trois programmes reflètent leurs postulats de base: les programmes Homebuilders prévoient un soutien rapide, centré sur le problème, avec des objectifs limités à atteindre ; dans le cas des programmes Families, les interventions sont plus longues et les objectifs dépassent la résolution d'une crise immédiate, tout comme les programmes Family Treatment, qui se rapprochent davantage des interventions de thérapie familiale à court terme.

Dans la prochaine partie de cet article, nous examinerons les principaux résultats présentés dans les recherches qui ont évalué l'efficacité de certains programmes de sauvegarde de la famille.

\section{L'EFFICACITÉ DES PROGRAMMES DE SAUVEGARDE DE LA FAMILLE}

On trouve deux générations d'études évaluatives portant sur les programmes de préservation ou de sauvegarde de la famille : les études qui ont été réalisées avant les années 90 et les études qui ont été entreprises depuis ce temps. Ces dernières se distinguent des premières surtout par le raffinement des concepts et des devis utilisés, ce qui confère davantage de validité et de sûreté aux résultats observés. C'est à la suite d'un bilan des premières recherches, effectué par Wells et Biegel et présenté à la conférence nationale sur les services de sauvegarde de la famille en 1989, que les grandes orientations de recherche de la nouvelle décennie ont été déterminées. Dans cette section, nous présenterons tout d'abord, à titre illustratif, les résultats généraux obtenus dans huit recherches évaluatives récentes ayant porté sur l'efficacité et I'utilité de ces programmes pour prévenir le placement des enfants et pour améliorer, de manière générale, le fonctionnement familial. Le choix de ces études s'est fait en raison de leur publication récente et de leur accessibilité. Il ne s'agit donc pas ici de faire état d'une recension exhaustive des écrits sur le sujet, mais plutôt de présenter les principaux thèmes évalués. En second lieu, nous résumerons les limites méthodologiques et conceptuelles de ces études et, en troisième lieu, nous formulerons quelques indications sur les 


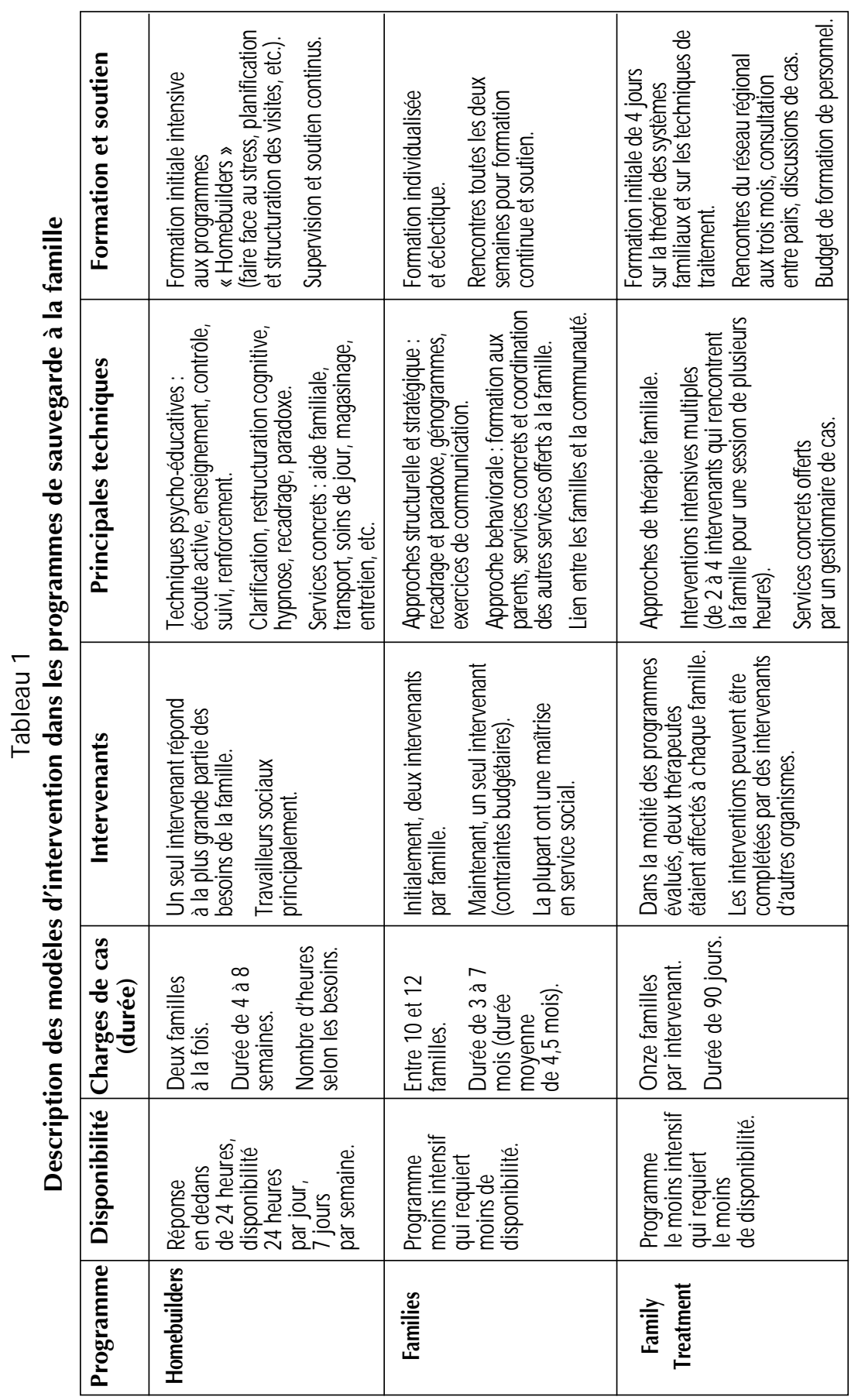


avenues de recherche qu'il y aurait lieu de poursuivre au moment de l'implantation de ces services.

\section{Efficacité des programme à prévenir le placement}

De manière générale, les programmes évalués semblent agir positivement sur la prévention du placement. II est cependant hasardeux d'indiquer un chiffre précis, puisque les définitions de ce concept varient trop d'une étude à une autre (nous reviendrons d'ailleurs plus loin sur cette question). II ressort tout de même des études évaluatives que ces programmes sont un peu plus efficaces que les services traditionnels (tableau 2). Feldman (1991) a constaté que I'application d'un programme appartenant au modèle des homebuilders avait permis de prévenir $93 \%$ des placements comparativement à $85 \%$ pour les services traditionnels. Ce pourcentage s'était cependant abaissé à $63 \%$ (contre $42 \%$ ), neuf mois après la fin de l'intervention. Kinney et al. (1990) ont obtenu, de leur côté, un taux de prévention de placement qui variait de $83 \%$ à $95 \%$ selon les caractéristiques des familles, tandis que Pecora et al. (1991) ont évalué qu'un programme de type homebuilders avait permis de prévenir $93 \%$ des placements à la fin du programme. Ce pourcentage n'était toutefois que de $67 \%$ douze mois plus tard, comparativement à $48 \%$ pour un petit groupe de contrôle ayant reçu des services traditionnels. En résumé, il ressort généralement des évaluations que ces programmes sont très efficaces à prévenir le placement (de $85 \%$ à $93 \%$ ), mais que leurs effets positifs diminuent avec le temps. Cette constatation amène les promoteurs de ces programmes à s'interroger sur la nécessité d'offrir des traitements répétés en vue d'améliorer les résultats. En effet, peut-être l'intervention de crise, à court terme, est-elle plus efficace, avec certaines clientèles, lorsqu'on la répète à quelques reprises.

Par ailleurs, Schwartz et al. (1991) ont observé, au moment de leur suivi, de 12 à 16 mois après l'entrée dans le programme, que $56 \%$ des adolescents qui avaient reçu des services d'un programme homebuilders avaient été placés, comparativement à $50 \%$ des adolescents du groupe de contrôle équivalent. Ces auteurs ont aussi noté que le nombre moyen de placements subis était relativement identique (2,5 placements par adolescent pour le groupe expérimental contre 2,3 placements par adolescent pour le groupe de contrôle). Ces deux groupes se distinguaient cependant sur la durée des placements. Les adolescents du groupe expérimental avaient été placés, au total, moins longtemps que les adolescents de l'autre groupe (4 777 jours c. 10037 jours). II ressort donc de cette étude que bien que les effets du programme sur la prévention du placement semblent s'amenuiser à moyen terme, celui-ci aurait un impact positif et réduirait la durée des placements en dehors du milieu familial. II s'agit cependant d'une hypothèse qui devra être examinée plus à fond, tout comme les effets de ces programmes sur des familles ayant des adolescents. 


\section{Facteurs associés à l'échec ou à la réussite de ces programmes}

Outre l'évaluation de la capacité de ces programmes à éviter le placement, certains chercheurs ont examiné les caractéristiques de la clientèle. Cet examen permet, notamment, de faire ressortir le profil des clients à qui cette forme $d$ 'intervention profite le plus.

Ainsi, Yuan et al. (1991) ont procédé à une analyse discriminante, afin de déterminer les facteurs permettant de distinguer les enfants qui avaient été placés et ceux qui étaient restés à domicile. Il ressort de cette analyse que le nombre de placements antérieurs, le placement pour motif de négligence, la menace d'abus à la fin du traitement et le fait qu'il y ait eu une enquête sur les possibilités d'abus ou de négligence après la mise en charge sont tous des éléments que l'on retrouve plus fréquemment chez les enfants placés. Nelson (1991a), de son côté, a aussi tenté de déterminer les facteurs de prédiction de l'échec d'un programme de sauvegarde de la famille. Elle a cependant développé deux modèles de prédiction selon le type de problèmes présentés par l'enfant. Il y aurait ainsi plus de risques de placement chez les enfants victimes d'abus ou de négligence lorsque les parents consomment de la drogue et que l'enfant reçoit des services communautaires de santé mentale. Du côté des jeunes contrevenants, les facteurs de prédiction les plus importants du placement seraient le fait d'avoir déjà vécu un placement et celui de fréquenter une école ordinaire. Finalement, Feldman (1991) a constaté un lien entre le placement de l'enfant et le score global à l'échelle de bien-être de l'enfant, à la sous-échelle " disposition parentale » et « performance de l'enfant ».

Deux éléments propres à la philosophie des programmes de sauvegarde de la famille ont été trouvés associés de manière importante à leur efficacité. Ainsi, Schwartz et al. (1991) ont observé que le degré d'engagement des clients et leur participation à la définition des objectifs du traitement ont pour effet de raccourcir la durée du placement quand ce dernier n'a pu être évité. Berry (1991), de son côté, a observé que le temps de service accordé au domicile de la famille a été le facteur de prédiction le plus important du maintien de l'enfant dans sa famille.

Enfin, Spaid et Fraser (1991) ont voulu savoir si le placement pouvait être plus facilement évité lorsque la situation problème était reliée à l'enfant plutôt qu'aux parents. Les résultats obtenus ont infirmé cette hypothèse. De plus, les auteurs en sont arrivés à subdiviser les familles composant leur échantillon $(n=453)$ selon deux types. Le premier comprend les familles où I'on observe des déficits parentaux : difficultés à répondre aux besoins des enfants et peu d'habiletés à superviser. Ces familles ont généralement des enfants plus jeunes. Le second type regroupe les familles où les déficits et les problèmes s'observent chez les enfants. Ces derniers seraient aussi plus âgés, en plus grande opposition à leurs parents, davantage portés à être impliqués dans des activités délinquantes et à consommer de la drogue. Le programme de traitement s'est révélé plus efficace auprès du premier type. 


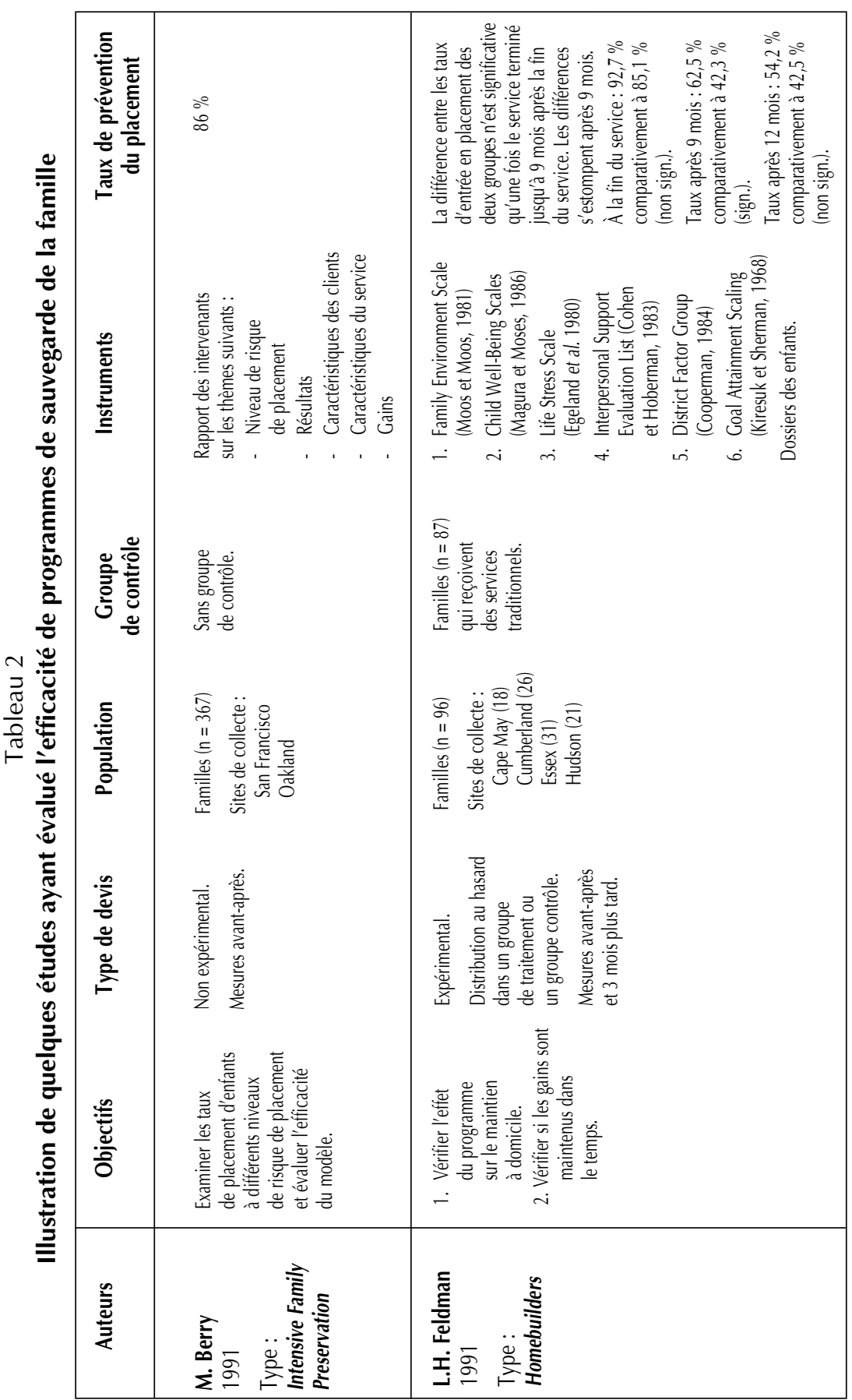




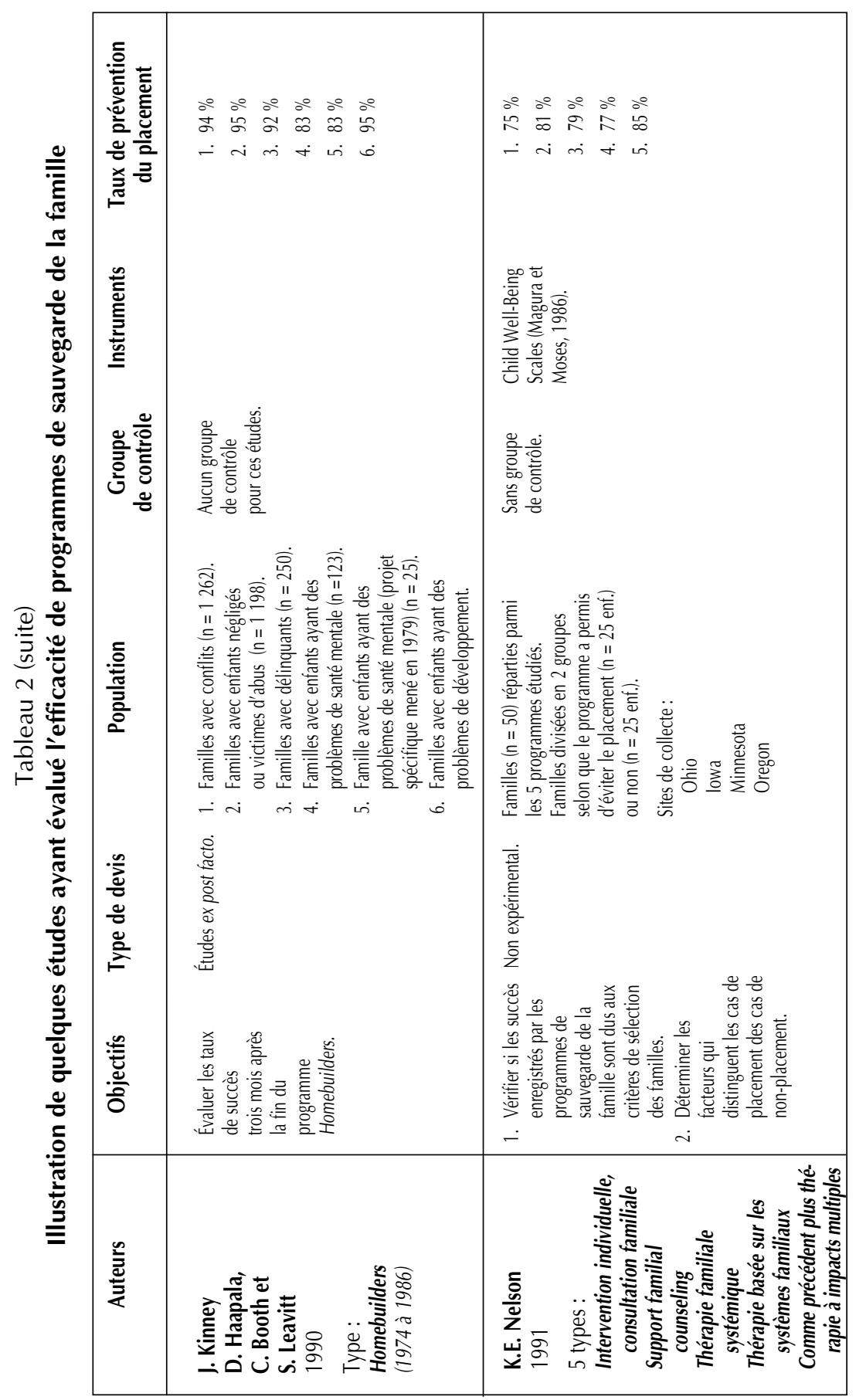




\begin{tabular}{|c|c|}
\hline 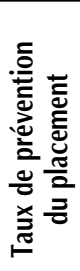 & 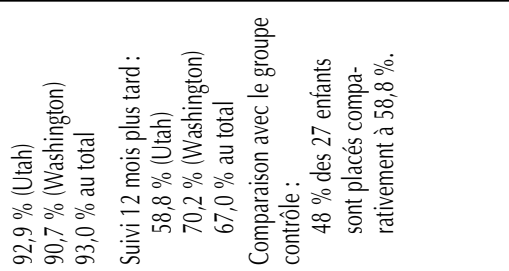 \\
\hline 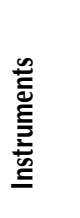 & 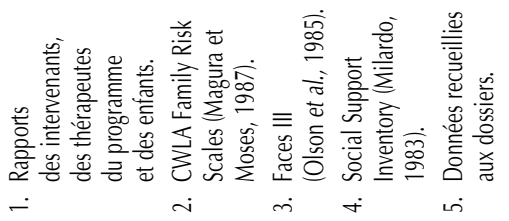 \\
\hline 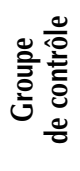 & 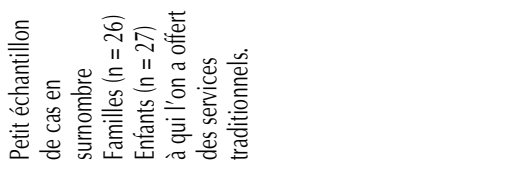 \\
\hline 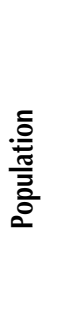 & 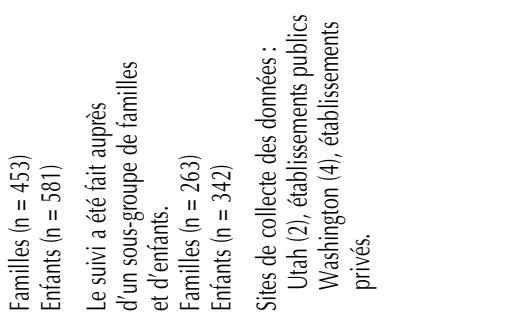 \\
\hline 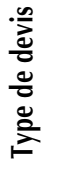 & 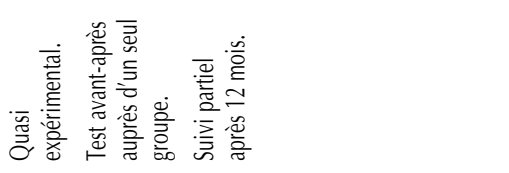 \\
\hline 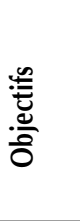 & 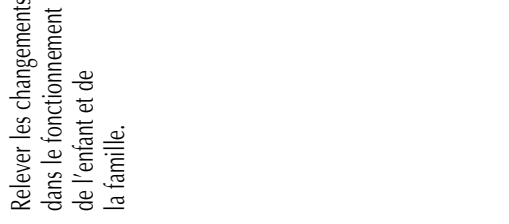 \\
\hline 竞 & 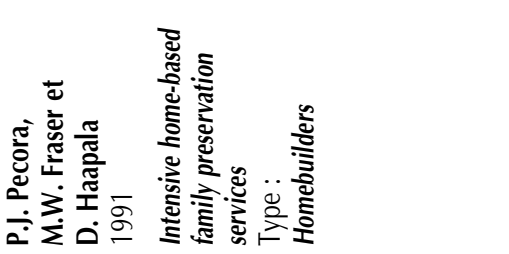 \\
\hline
\end{tabular}




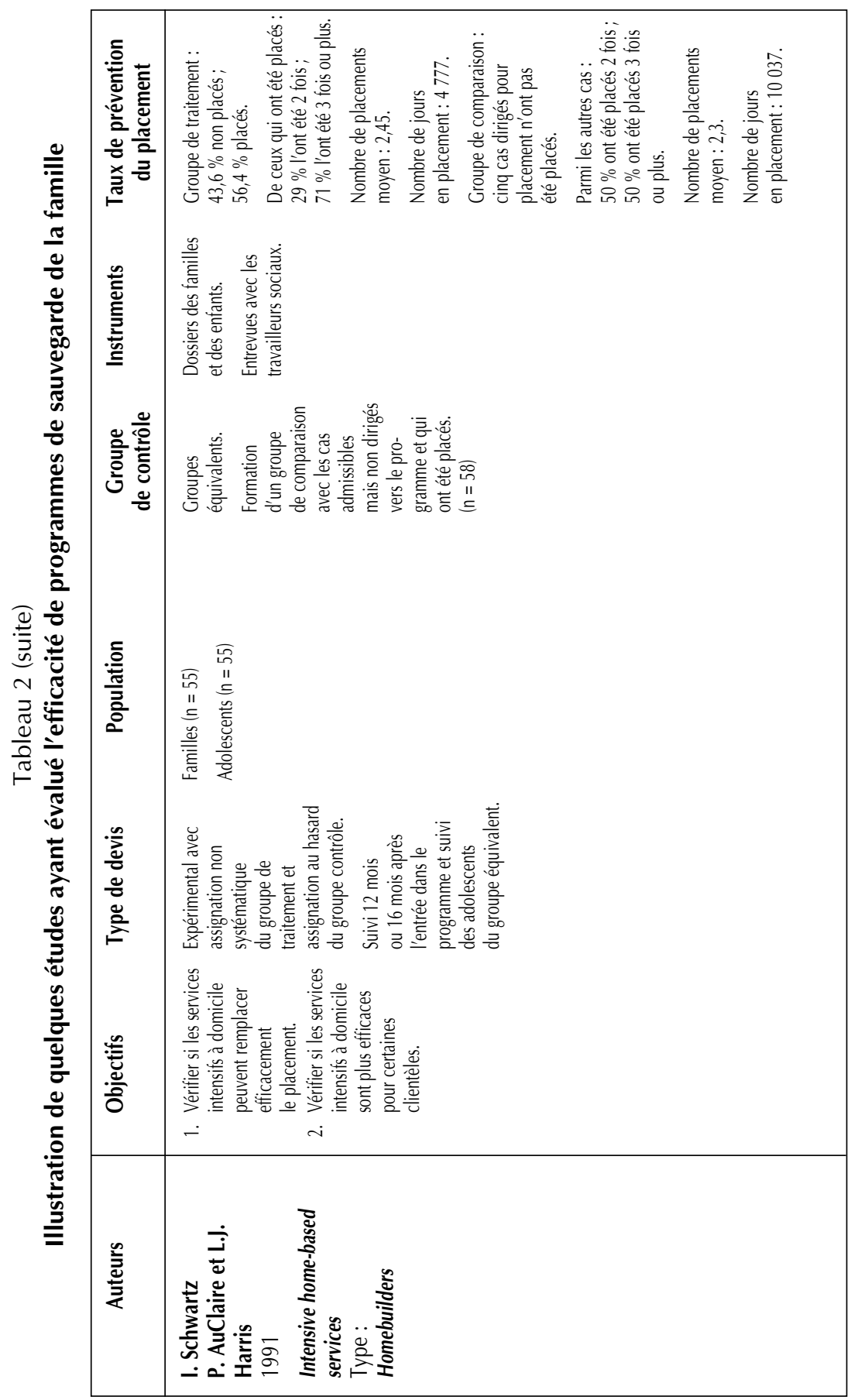




\begin{tabular}{|c|c|}
\hline 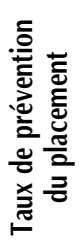 & 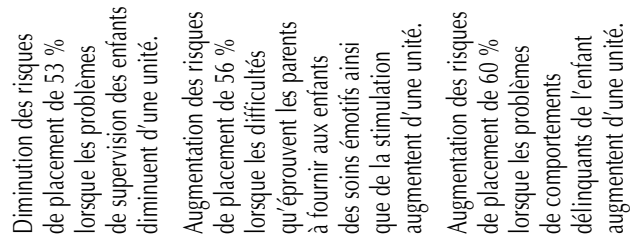 \\
\hline 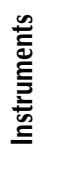 & 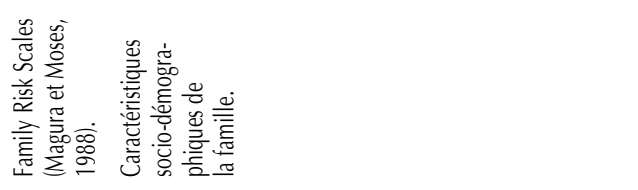 \\
\hline 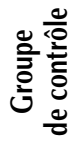 & 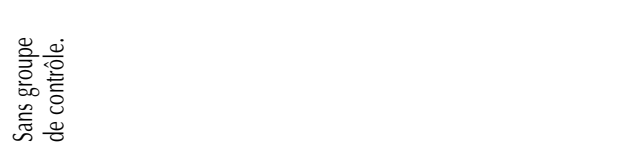 \\
\hline 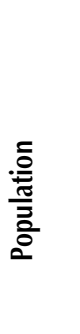 & 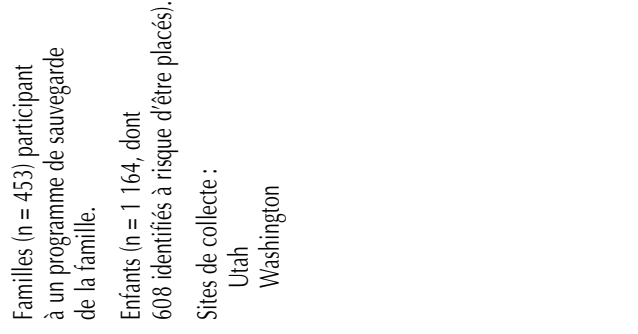 \\
\hline 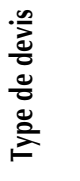 & 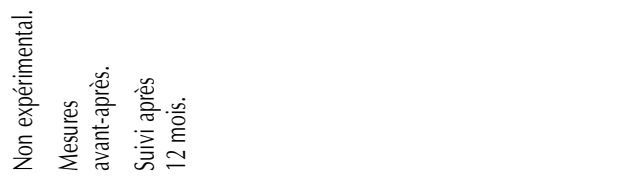 \\
\hline 产 & 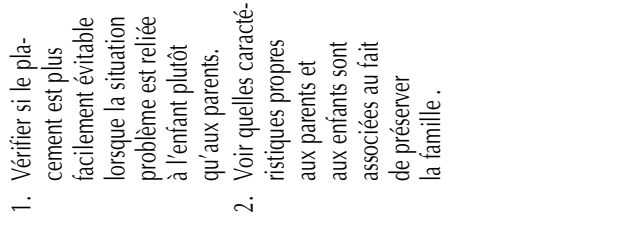 \\
\hline 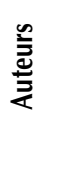 & 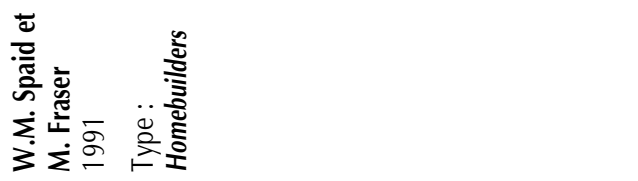 \\
\hline
\end{tabular}




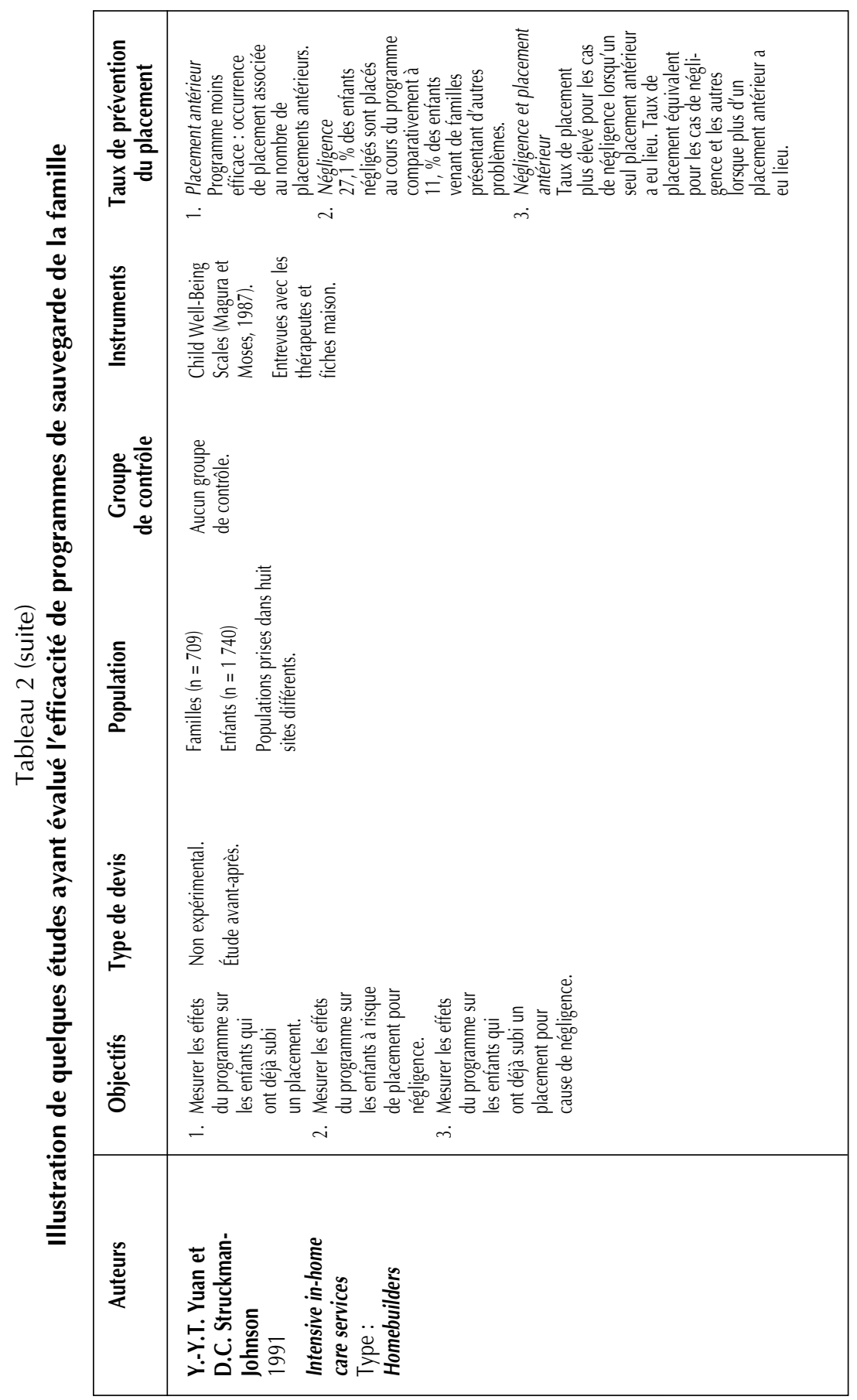




\section{Autres effets des programmes}

Outre l'objectif ultime de ces programmes, qui est d'éviter le placement de l'enfant, quelques chercheurs ont voulu évaluer leurs effets sur d'autres variables que le maintien de l'enfant dans son milieu naturel. À ce chapitre, Feldman (1991) a observé, pour un même groupe de familles, des changements statistiquement significatifs, entre le début du programme et trois mois après sa fin, à 10 des 18 échelles (voir tableau 2) utilisées visant à mesurer le soutien, le fonctionnement familial et le bien-être de l'enfant. Par ailleurs, des différences statistiquement significatives entre le groupe expérimental et le groupe de contrôle n'ont été observées qu'à deux échelles, soit celle portant sur l'environnement familial (item « intellectuel-culturel ») et celle mesurant le bien-être de l'enfant (item "disposition parentale »). Dans les deux cas, les familles du groupe expérimental avaient un meilleur fonctionnement à ces items que celles du groupe de contrôle. Pecora et al. (1991) avaient déjà observé, pour leur part, que les changements positifs dans le fonctionnement familial de l'enfant et de la famille étaient associés au succès du programme mesuré par l'absence de placement à la fin du traitement.

Kinney et al. (1991) ont aussi évalué les principales améliorations perçues par des intervenants et des parents engagés dans les programmes de sauvegarde de la famille. Ainsi, les répondants ont indiqué percevoir une amélioration de $100 \%$ des problèmes affectifs, de désorientation, d'hallucinations, de manque de motivation, de dépendance, d'agression sexuelle, des désordres de la pensée, des désordres affectifs, des obsessions et des phobies. Une amélioration allant de $90 \%$ à $100 \%$ a été observée dans les situations d'agression envers les autres, de manque de coopération et de colère. La dépression, l'hyperactivité, les mauvais jugements et les problèmes de communication se seraient améliorés de $80 \%$ à $90 \%$. Enfin, l'absentéisme scolaire, l'anxiété, le manque de contrôle des impulsions et le vandalisme se seraient améliorés de $70 \%$ à $80 \%$. On note, à l'autre extrémité, peu d'amélioration (de $20 \%$ à $30 \%$ ) dans les cas d'abus d'alcool et de problèmes d'apprentissage.

La présentation de ces quelques données démontre que les services offerts dans le cadre des programmes de sauvegarde de la famille donnent généralement des résultats positifs, tant sur le plan de la prévention du placement que sur le plan de l'amélioration du fonctionnement familial. Toutefois, force est de constater que ces études évaluatives ont d'importantes limites nous empêchant de généraliser les résultats et de conclure à l'efficacité plus grande d'un type de services plutôt que d'un autre. 


\section{LES LIMITES MÉTHODOLOGIQUES DES ÉTUDES ÉVALUATIVES}

Dans cette partie, nous traiterons différents éléments méthodologiques qui invalident, dans certains cas, ou réduisent, dans d'autres, la portée des résultats obtenus dans les études évaluatives.

\section{Les protocoles d'évaluation}

La première génération d'études évaluatives des programmes de sauvegarde de la famille, qui s'étend jusque vers la fin des années 80 , comporte des failles méthodologiques suffisamment importantes pour remettre en cause les conclusions avancées au sujet des effets réels de ces programmes. Depuis, les chercheurs ont amélioré certains aspects de leurs protocoles, mais on recense encore de nombreuses lacunes qui rendent impossible un jugement définitif sur l'efficacité des programmes.

Les premières évaluations, réalisées au cours des années 1980-1985, ne comportaient pas de groupes de comparaison et étaient généralement effectuées ex post facto (W. Showell et H. R. Hartley, cités dans Frankel, 1988 ; Leeds, 1984 ; Landsman, 1985) ; on y mesurait le taux de placement d'une population soumise à un programme et, dans certains cas, l'amélioration du fonctionnement familial à la fin du programme. En l'absence de mesure de départ et de groupe de contrôle, il était impossible de démontrer que les effets obtenus étaient réellement attribuables au programme. Les études plus récentes ont parfois comblé cette lacune en perfectionnant les devis de recherche et en prévoyant des groupes de comparaison ou des groupes de contrôle avec distribution au hasard des sujets (Feldman, 1991 ; Pecora, Fraser et Haapala, 1991, Schwartz, AuClaire et Harris, 1991). Malheureusement, les services offerts aux familles des groupes de contrôle sont peu décrits et rien ne permet de conclure qu'ils se distinguent vraiment des services offerts aux groupes expérimentaux. Les évaluations des programmes de sauvegarde de la famille sont aussi avares de descriptions concernant les méthodes et les contextes de collecte des données et les caractéristiques de ceux qui ont recueilli les informations. Enfin, Rossi (1992) dénonce la simplicité des stratégies d'analyse et l'étroitesse des objets d'étude. Il faudrait étendre l'analyse à $\mathrm{d}^{\prime}$ autres effets possibles des programmes, par exemple à la réduction de l'abus et de la négligence, à la modification des comportements familiaux et à la sécurité future des enfants des familles dirigées vers les programmes de services intensifs. Comme nous avons pu le constater dans la partie précédente, cependant, les évaluations les plus récentes ont élargi leurs objets d'étude pour inclure le fonctionnement familial (Pecora et al., 1987), tandis que d'autres ont examiné certaines caractéristiques des clientèles associées au 
succès ou à l'échec des programmes (Spaid et Fraser, 1991 ; Nelson, 1991a ; Schwartz et al., 1991 ; Yuan et Struckman -Johnson, 1991).

\section{Les critères de sélection des populations étudiées et l'importance des échantillons}

La majorité des études évaluatives ont analysé des programmes dont le principal critère de sélection des familles était le « risque imminent de placement » d'un enfant ; or, plusieurs définitions du « risque imminent » ont été formulées; certains auteurs ont retenu le niveau de pauvreté, la monoparentalité et la présence d'adolescents (Kinney et al., 1977 ; Landsman, 1985) ; d'autres se sont fiés au jugement de l'intervenant quant au risque de placement dans les 24 ou 48 heures (Pecora et al., 1987) ; d'autres encore ont considéré que l'enfant était « à risque de placement » $s^{\prime} i l$ avait déjà été placé, s'il était victime d'abus ou s'il présentait des troubles de comportement (Berry, 1991). On observe de plus que les administrateurs ont fréquemment assoupli les critères d'admission en cours d'application du programme, afin d'augmenter le nombre de participants admissibles. L'absence de rigueur, la multiplicité et la subjectivité des définitions du « risque imminent de placement » constituent donc des problèmes majeurs dans l'évaluation de ces programmes.

Afin de pallier cette difficulté, quelques chercheurs ont tenté d'évaluer la capacité d'instruments de mesure à déterminer l'imminence du risque de placement. Nelson (1991b) a en effet évalué la possibilité d'utiliser la Child Well-Being Scales (Magura et Moses, 1986) pour prédire le placement d'enfants. Les familles ( $n=752$, dont 219 présentaient un problème d'enfants maltraités) ont été recrutées parmi six programmes de sauvegarde de la famille. Deux sous-échelles de la CWBS (échelles portant sur les parents et les enfants) ont été administrées avant et après le programme. L'auteure en arrive à la conclusion que bien que l'instrument permette de documenter les changements survenus dans le fonctionnement familial, il ne constitue pas, dans sa forme actuelle, une mesure adéquate pour prédire le placement ou non des enfants.

Thieman et Dall (1992) ont, de leur côté, cherché à vérifier la capacité de l'instrument Family Risk Scales (Magura, Moses et Jones, 1987) à prédire I'imminence du risque de placement. L'étude a été menée auprès de 995 familles divisées en deux groupes, selon le résultat obtenu (bas ou élevé) à cette échelle, au temps « avant » du programme. Les résultats obtenus, au temps « après » du programme, ont forcé les chercheurs à conclure que l'instrument n'est pas très utile dans la prédiction de la possibilité de placement de l'enfant, même s'il permet d'évaluer les modifications dans le fonctionnement familial. Aussi faut-il conclure qu'aucun instrument de mesure ne permet à I'heure actuelle d'évaluer de manière objective le risque de placement. 
Outre les problèmes de définition du « risque de placement » comme critère $d$ 'acceptation, les études évaluatives des programmes de sauvegarde offrent peu d'informations sur les familles et les enfants qui sont admis au programme (Jones, 1985), sur ceux qui ont refusé d'y participer ou qui se sont désistés en cours de programme. Ces informations permettraient de mieux comparer les études entre elles. Elles énoncent aussi divers critères d'exclusion de clientèles (par exemple, les familles d'accueil de la parenté ou les placements à court terme) qui limitent les possibilités de généralisation des conclusions (Leeds, 1984 ; Pecora, Fraser et Haapala, 1991). Rossi (1992) note enfin que, malgré le grand nombre de sous-groupes de familles auprès desquelles les programmes ont été appliqués, le nombre de familles de chaque sous-groupe est trop faible pour que l'on puisse réaliser les analyses pertinentes à leur comparaison sur les variables importantes d'un même programme.

\section{Les programmes}

Même si les résultats des évaluations semblent démontrer que ces programmes, pris individuellement, ou regroupés selon les divers modèles (Homebuilders, Families, Family Treatment) ont pu éviter des placements (Nelson, Landsman et Deutelbaum, 1990) ou améliorer le fonctionnement des enfants et des parents qui ont bénéficié des services (Pecora et al., 1991 ; National Resource Center on Family-Based Services, 1989 ; Kinney et al., 1990), on note une telle variété de critères de sélection, de clientèles retenues (âge des enfants, problématique) et de conditions d'application qu'il est impossible d'en tirer des conclusions qui s'appliqueraient à un ensemble de programmes.

De façon générale, les programmes sont peu et mal décrits et ne présentent pas les contenus théoriques, les techniques de traitement, le personnel mis à contribution et les ressources utilisées. En l'absence de tels descripteurs, il est difficile de comparer l'efficacité des divers modèles et de généraliser l'efficacité d'un type de programme en particulier. Il apparaît enfin que les évaluations de ces programmes ont débuté beaucoup trop tôt après le début de leur implantation (Rossi, 1992). Il faudrait prévoir un temps d'ajustement du programme de manière que l'évaluation porte sur des éléments dont la stabilité est assurée tout au long du processus.

\section{L'interprétation des résultats}

La majorité des études évaluatives des programmes de sauvegarde de la famille ont utilisé comme mesures d'efficacité de ces programmes la prévention du placement, I'amélioration du comportement des enfants et de leurs 
parents et la réduction des coûts des services. Nous présentons ici les principales limites et difficultés observées en rapport avec le choix de ces mesures comme indicateurs de succès.

\section{Les taux de placement}

L'utilisation du taux de placement (ou de non-placement) comme mesure d'efficacité pose certains problèmes (Wells et Biegel, 1989 ; Feldman, 1991 ; Yuan et Struckman-Johnson, 1991 ; Rossi, 1992). Premièrement, on ne peut prétendre que tous les placements qui ont été évités se seraient produits si les familles n'avaient pas été dirigées vers le programme. Deuxièmement, le placement peut être considéré à la fois comme résultat du programme et comme traitement. Dans certains cas, en effet, il peut être souhaitable qu'un enfant soit placé ; il est alors évident qu'on ne peut considérer ce placement comme un échec du programme. Troisièmement, le placement ou non d'un enfant peut être fonction de la disponibilité des ressources ; un placement évité par manque de ressources de placement ne peut être considéré comme un effet du programme. Quatrièmement, l'absence de placement peut signifier tout simplement que la situation ne requiert plus cette mesure, l'urgence étant passée. Enfin, on note un manque d'uniformité dans les définitions de placement : par exemple, certains auteurs éliminent les placements d'urgence de quelques heures ou les placements dans le milieu naturel de l'enfant (oncle, tante, grands-parents, etc.), alors que d'autres les incluent dans leurs situations de placement (Berry, 1991).

\section{Le fonctionnement de l'enfant et de sa famille}

Les études évaluatives plus récentes, qui ont procédé à partir de devis expérimentaux ou quasi expérimentaux, ont éliminé une partie des problèmes de mesure de l'amélioration du fonctionnement de l'enfant et de sa famille en y introduisant, entre autres, une panoplie d'instruments validés (Feldman, 1991 ; Pecora, Fraser et Haapala, 1991). Cependant, comme plusieurs de ces instruments sont administrés par les intervenants eux-mêmes et que l'on observe un fort taux de roulement de personnel au moment de l'application des programmes, il arrive que le fonctionnement ne soit pas évalué par les mêmes intervenants avant et après le traitement, ce qui introduit un problème de fiabilité des résultats (National Resource Center on Family-Based Services, 1989). Comme dans toutes les études de ce type, il est aussi très difficile de contrôler tous les paramètres qui peuvent avoir eu un impact sur les comportements des personnes au cours du traitement. Soulignons cependant que l'on cherche à améliorer les études qui ont pour objet le fonctionnement de l'enfant et de sa famille (Thieman et al., 1990 ; Nelson, 1991b ; Feldman, 1991 ; Yuan et Struckman-Johnson, 1991) et qu'il faudra vérifier plus avant si les 
situations d'abus ou de négligence ont diminué, si l'amélioration des comportements observée est permanente et si l'on augmente les compétences parentales de façon significative.

\section{L'analyse des coûts}

Les études des coûts des services offerts dans les programmes de sauvegarde de la famille et des économies qu'ils permettent de réaliser ne sont pas encore concluantes, parce qu'elles n'ont pas considéré un certain nombre d'éléments. Premièrement, tant que l'on ne pourra pas certifier que le placement a été évité grâce au programme, il sera difficile de lui en attribuer l'économie. Deuxièmement, les évaluations n'ont pas considéré, dans les coûts, I'ensemble des services qui ont pu être offerts à la famille avant et après le programme, ces services ayant pu varier grandement en qualité et en quantité (Frankel, 1988). Troisièmement, certaines études ont comparé le coût du placement de tous les enfants du programme dans des ressources du même type, généralement les plus chères, avec le coût du maintien à domicile (Magura, 1981) ; cette façon de faire présuppose que tous les enfants auraient été placés s'ils n'avaient pas bénéficié des services et que des ressources de même nature auraient été utilisées pour tous les enfants. Quatrièmement, dans leurs projections, les évaluateurs ont supposé que les taux de placement demeureraient constants dans le temps ; or, on n'a qu'à regarder les statistiques les plus récentes pour reconnaître que ces taux fluctuent au fil des ans (Family Impact Seminar, 1990). Enfin, il faut souligner que les évaluations n'ont pas tenu compte des coûts de formation du personnel ou de restructuration des services (Frankel, 1988) au moment de l'implantation des programmes.

\section{Les avenues de recherche}

Même si les études évaluatives des programmes de sauvegarde de la famille comportent de nombreuses limites, il faut reconnaître que le domaine est jeune et que les recherches les plus récentes présentent déjà un " produit » amélioré. Par ailleurs, il ne faut pas perdre de vue que l'évaluation de programmes auprès d'une population à risque pose des problèmes d'éthique et de mise en œuvre que l'on ne peut sous-estimer. II n'en demeure pas moins, cependant, que les efforts des chercheurs dans le domaine des services de sauvegarde de la famille doivent être poursuivis. On peut cependant profiter de ces premières constatations pour mieux cibler les recherches futures. Nous énumérons ci-après les principales orientations de recherche découlant de I'analyse des évaluations des programmes de sauvegarde de la famille :

1) déterminer, parmi la population d'enfants placés, combien répondraient aux critères actuels d'admission dans les programmes de sauvegarde de la famille ; 
2) prévoir des protocoles expérimentaux pour analyser les effets des programmes, non seulement sur la prévention du placement, mais aussi sur tous les autres aspects du fonctionnement de la famille et de l'enfant ;

3) réaliser des études longitudinales pour examiner si les acquis se maintiennent dans le temps ;

4) intégrer aux études les aspects environnementaux, c'est-à-dire les caractéristiques du système de services, les réseaux informels des familles et les caractéristiques des régions ou communautés, éléments qui peuvent avoir une influence sur les effets des programmes ;

5) évaluer l'application du programme et de ses principes sous-jacents, les difficultés rencontrées, les effets non prévus, la satisfaction des clients ;

6) évaluer le fonctionnement des enfants et des familles à la fin du programme ;

7) évaluer les coûts des services offerts pendant le programme et après le programme ;

8) examiner les éléments qui peuvent faciliter ou au contraire empêcher la mise en place de tels programmes dans d'autres contextes (Wells et Biegel, 1989).

On peut aussi souhaiter le développement d'outils de recherche qui rendent possible une évaluation objective et plus standard de l'imminence du risque de placement.

\section{LES CONDITIONS D'IMPLANTATION DES PROGRAMMES}

La première partie de cet article a présenté les programmes de sauvegarde de la famille en insistant surtout sur la philosophie et sur les valeurs qui sont véhiculées dans ces programmes et sur l'importance de respecter certains principes de base au moment de l'intervention. La seconde partie a fait état des évaluations des résultats de ces programmes en mettant en évidence les taux de réussite dans la prévention du placement et l'amélioration du fonctionnement de l'enfant et de sa famille, mais en soulignant aussi les limites de ces évaluations. Ces programmes semblent prometteurs, mais, selon les chercheurs, il est encore trop tôt pour généraliser les résultats sur l'efficacité de ces programmes dans la prévention du placement.

Cependant, même si les programmes qui ont été évalués ne peuvent être comparés quant à leur clientèle, aux critères d'admissibilité ou aux définitions de placement retenues, nous n'avons pas repéré d'études contredisant les résultats d'une efficacité certaine à réduire les taux de placement. Les limites présentées plus tôt se rapportent davantage aux évaluations de ces programmes qu'à leur fonctionnement lui-même. C'est dans cette perspective que nous présentons maintenant quelques facteurs de réussite liés à l'implantation et au fonctionnement des programmes de sauvegarde de la famille. 


\section{Partager la philosophie de base}

L'implantation de programmes de sauvegarde de la famille exige $\mathrm{d}^{\prime}$ abord que l'on s'assure que ceux et celles qui soutiendront et appliqueront les programmes adhèrent vraiment à leur philosophie. Dans un contexte social où la protection de l'enfant est une valeur importante, les principes énoncés plus tôt (le respect des familles, la reconnaissance des forces et des habiletés, etc.) doivent se concrétiser dans des attitudes, des valeurs et des comportements précis auprès des familles en difficulté. Par ailleurs, dans un système de distribution de services où les mandats des acteurs (dans les établissements surtout) sont passablement "étanches », les responsables de I'implantation de ces programmes doivent travailler dans des perspectives de continuité et de souplesse entre les services :

In the specific case of family preservation, care must be taken to ensure that policy initiatives proceed from the notion of a continuum of needed services to avoid the myopia that has sometimes accompanied other service initiatives (Whittaker, 1991, p. 298).

Enfin, la philosophie de base doit avoir été adoptée par toutes les instances du réseau, ministère, conseil d'administration, directeur général, cadres supérieurs et intermédiaires. C'est là une condition essentielle à la survie de ces programmes.

\section{Définir les critères d'admissibilité}

On a vu, dans la description des résultats, que la sélection des familles admissibles à de tels services et la précision du moment où elles le deviennent constituent des éléments critiques au moment de l'implantation des programmes. Est-ce que la sécurité de l'enfant est compromise ? Est-ce qu'un service est requis ? Est-ce qu'il y a un risque immédiat ou imminent de placement d'un enfant de la famille? Est-ce qu'une intervention moins intensive pourrait suffire? Est-ce que cette intervention a été offerte, mais n'a pas donné les résultats attendus ? Est-ce que la famille est un endroit sécuritaire pour tous les membres de la famille et pour l'intervenant ? Est-ce que la famille peut profiter de ces services? Est-ce que les membres de la famille sont d'accord pour s'engager dans un processus de changement et aptes à le faire?

En fait, les critères d'admissibilité vont varier selon la philosophie du programme, les ressources humaines disponibles, les ressources financières, l'expérience et l'expertise des intervenants et l'éventail de services auxquels ceux-ci peuvent recourir dans l'environnement des familles. À ces critères " d'entrée » dans le programme s'ajouteront des critères « de sortie » du programme (durée des services, durée du suivi) et des critères « de retour » dans le programme. 
Enfin, plusieurs auteurs ont mis en évidence que le processus de prise de décision clinique n'est pas uniforme et donne des résultats différents d'un décideur à l'autre. Il devient donc très important dans I'implantation de ce type de programme que les règles qui président aux prises de décision soient le mieux définies possible.

\section{Définir la population cible}

Le choix des familles qui font partie des programmes de sauvegarde de la famille est crucial. On observe, à l'intérieur des programmes actuellement en place dans les services sociaux, un éventail impressionnant de problèmes : des problèmes d'abus, de négligence, de relations parents-enfants, d'agressivité, de consommation de drogues et d'alcool, des problèmes environnementaux, etc. Il semble, d'après les études, que les programmes de sauvegarde de la famille soient tous efficaces, à des degrés divers, peu importe le type de problème. Il ne s'agit donc pas de déterminer quels problèmes ont les plus grandes chances de profiter de ce type de services, mais il faut plutôt se demander si, dans une situation donnée, I'intervention de crise à court terme peut donner des résultats.

Il faut aussi se demander si ce type de services ne devrait pas s'adresser qu'à des nouveaux cas ou à des cas qui sont dans les systèmes de services sociaux depuis peu de temps. L'expérience du New Jersey est intéressante sur ce plan, parce qu'elle démontre qu'il est possible d'élargir l'éventail de la clientèle admissible au fur et à mesure que les membres de l'équipe prennent de l'expérience dans le type d'interventions et dans la connaissance de l'environnement des familles (Feldman, 1991).

\section{Recruter, superviser, former}

Un des critères de succès de l'implantation des programmes de sauvegarde de la famille se rapporte au recrutement des divers intervenants qui doivent endosser la philosophie et les objectifs du programme. II faut aussi tenir compte de ces aspects au moment du remplacement du personnel, puisque les évaluations démontrent que le roulement de personnel peut devenir un facteur clé du succès ou de l'échec de ces programmes

On doit aussi insister pour que des sessions de formation et de supervision soient planifiées : une formation initiale, régulièrement mise à jour en fonction des besoins que soulèvent les expériences concrètes des intervenants. Un tel processus permet non seulement l'acquisition de nouvelles idées et habiletés, mais soutient I'intervenant et l'aide à assumer les tensions inhérentes à ce type d'intervention. II renforce aussi I'adhésion à la philosophie et aux valeurs prônées par les programmes de sauvegarde de la famille (Blythe et al., 1992). 
Les conditions de la supervision offerte aux intervenants doivent aussi refléter les principes et la philosophie des programmes, c'est-à-dire que les superviseurs doivent être disponibles, capables d'utiliser les crises pour venir en aide et surtout bien centrés sur les besoins de chaque intervenant. L'utilisation de moyens non traditionnels de supervision, comme les téléphones en soirée, la supervision par les pairs et la tenue de rencontres brèves mais régulières constituent autant de moyens qui assurent aux intervenants un soutien constant et adapté au type d'intervention de ces programmes.

Blythe et ses collègues (1992) suggèrent deux types de rencontres de supervision : premièrement, des rencontres où les cas sont révisés et où la discussion des objectifs est complétée par l'expression des difficultés et des frustrations rencontrées au cours du processus d'aide à la famille ; deuxièmement, des rencontres où sont discutées les diverses dimensions du programme et où toutes ses composantes peuvent être remises en question.

Enfin, les superviseurs et les gestionnaires doivent pouvoir compter sur un soutien de leur établissement, en accord avec leur mandat et avec les fonctions de mobilisation et de liaison avec l'environnement extérieur qu'exigent ces programmes de sauvegarde de la famille.

\section{Assurer la sécurité du personnel}

Comme on l'a vu à la section portant sur la philosophie et les principes, une des principales caractéristiques des programmes de sauvegarde de la famille est d'offrir des services dans le milieu familial et dans son environnement. Les intervenants doivent donc pouvoir compter sur des mécanismes de prévention de la violence en tout temps, sur des processus de déclaration, rapides et simples, de situations violentes ou potentiellement violentes, sur des services de protection concrets et des services d'aide personnelle en cas de manifestations de violence.

\section{Ajuster les tâches et les horaires de travail}

Par leur nature même, les programmes de sauvegarde de la famille comportent une grande variété de tâches. En fait, I'accent est mis sur les résultats à atteindre avec la famille, et le choix des meilleurs moyens pour y arriver dans les meilleurs délais est laissé à l'intervenant. Ceux et celles qui acceptent de participer à ce type de programme ne doivent pas compter sur une description de tâches traditionnelle, mais doivent ajuster leurs interventions dans chaque famille aux mandats et aux objectifs qu'ils poursuivent en collaboration avec les autres partenaires de l'intervention. Comme l'intervenant a peu de temps pour réaliser ses objectifs, qu'il doit être disponible rapidement et qu'il doit livrer les services à domicile, I'horaire « 9 à 5 » avec 
un peu de temps supplémentaire à l'occasion ne convient pas à ce type de programme. Les politiques relatives aux heures de travail doivent donc permettre à l'intervenant d'être flexible et de varier son horaire de travail en fonction des besoins, tout en lui accordant le répit nécessaire à un travail aussi exigeant. Ainsi une organisation qui demande une semaine de travail de 32 heures et demie doit accepter qu'un intervenant répartisse sur quelques semaines les heures supplémentaires travaillées. On comprend alors que dans une même organisation certains intervenants auront un horaire plus souple, alors que d'autres, à l'intérieur de services réguliers, continueront de fonctionner selon un horaire fixe.

De plus, ce type d'intervention commande une diminution du temps consacré à des tâches administratives, à l'application de procédures et aux activités d'identification et de prise de contact avec les ressources du milieu. On pourrait envisager une collaboration entre divers intervenants sur ces aspects.

\section{La transparence dans les relations avec l'environnement interne}

L'implantation des programmes de sauvegarde de la famille par des organisations qui sont déjà engagées dans la distribution de services (pensons par exemple aux Centres locaux de services communautaires, aux Centres de protection de l'enfance et de la jeunesse ou aux Centres de réadaptation pour jeunes en difficultés d'adaptation) obligera à remettre en question les valeurs, les objectifs, les modalités de fonctionnement, les politiques et procédures des modules déjà en place.

La gestion des interfaces avec les programmes de services existants (orientation et transfert de clientèles entre modules ou services à l'interne, demande de services cliniques spécialisés, procédures d'utilisation de familles d'accueil ou de ressources intermédiaires, etc.), avec les groupes mandatés pour représenter les membres de l'organisation (dirigeants syndicaux, association de cadres, conseil consultatif du personnel clinique, conseil consultatif du personnel administratif, comité de la condition féminine, etc.), avec les divers services de soutien de l'organisation (conseillers juridiques, systèmes d'information, etc.) doit être une préoccupation constante. Chacun doit connaître les exigences de celui qui le précède et de celui qui le suit, afin de «bien faire du premier coup ».

\section{Une grande complicité avec les ressources de la communauté}

Les programmes de sauvegarde de la famille font appel à une grande variété de services offerts dans la communauté afin d'apporter aux familles en difficulté I'aide dont elles ont besoin. Il est démontré que l'efficacité de ces 
programmes réside en partie dans la mise à contribution de toutes les ressources existantes ; il ne s'agit pas simplement d'en faire le relevé, mais d'établir des contacts qui amélioreront l'accessibilité aux services et la rapidité avec laquelle on pourra répondre aux besoins des familles. Ces démarches administratives doivent être faites et bien faites. Il s'agit de préparer le terrain, de simplifier les procédures et de faire connaître les intentions.

Soulignons, en terminant, que les services intensifs auprès des familles ne sont habituellement pas les seuls services offerts aux familles. On observe en effet, dans les programmes américains, que des liens doivent être établis entre les services dits de crise, les services qui ont précédé et ceux qui suivront. Les gestionnaires de ces programmes doivent donc connaître les autres programmes de services ainsi que leurs critères d'accès et tenir cette information à jour, établir des procédures formelles pour faciliter l'accès à ces programmes et les inclure dans des arrangements écrits et personnalisés et, enfin, stimuler la création de ressources nécessaires non encore existantes.

Nous présentons, en terminant cette partie, quelques recommandations, inspirées en partie de Whittaker (1991), relatives à l'implantation d'un service de sauvegarde de la famille.

1) S'assurer de la concordance entre le programme implanté et les intentions de départ.

2) Ne pas exclure a priori certaines clientèles, mais plutôt se demander comment intégrer les diverses composantes du programme pour mieux leur venir en aide.

3) Ne pas toujours "réinventer la roue ", mais adapter à ses besoins propres des expériences qui ont fait leurs preuves.

4) Favoriser l'innovation.

5) Ne pas se contenter de donner beaucoup de services concrets aux familles sans vraiment savoir si ce que l'on fait donne les résultats attendus et sans comprendre pourquoi on réussit et pourquoi on échoue.

6) Les programmes d'aide intensive à la famille ne font pas de miracles. En rester conscient et garder en tête que ce qui compte, c'est de donner le bon service à la bonne famille.

\section{CONCLUSION}

Les programmes de sauvegarde de la famille ont d'abord été mis en place pour prévenir le placement d'enfants en dehors du milieu familial. D'après les études effectuées, ces programmes semblent prometteurs, tant par les taux de prévention du placement qu'ils permettent d'atteindre que par leur capa- 
cité à améliorer le fonctionnement des familles et des enfants. La philosophie de ces programmes, la rapidité d'intervention, l'intensité des services, l'apport de services concrets et l'intervention en milieu familial sont des éléments clés de leur succès. Ces services permettent aussi de mettre l'accent sur les capacités des familles plutôt que sur leurs limites. On peut dire de ces programmes qu'il s'agit d'un type de services précis et d'une toute nouvelle approche qui nécessite non seulement la mise en place de nouveaux services, mais une restructuration complète du système de services. Ces programmes proposent une nouvelle vision des familles, une nouvelle conception des services, de nouvelles méthodes de distribution des services et de nouveaux modes de coopération avec les autres organismes du milieu.

Il va de soi, bien que la question n'ait pas été abordée dans cet article, que la formation des intervenants et, par la suite, leur supervision sont des éléments très importants de ces programmes. Il faut en effet reconnaître que I'application de cette forme d'intervention appelle à une modification en profondeur des pratiques actuelles. C'est donc par la formation et la supervision que devra être transmise cette nouvelle philosophie de services qui modifie notre façon de voir la famille ainsi que notre manière de rendre les services. On ne devra jamais perdre de vue que la compétence des intervenants et leurs habiletés à utiliser les éléments fondamentaux des programmes de sauvegarde sont la principale garantie de l'efficacité de cette nouvelle approche. Par ailleurs, et comme le lecteur aura pu le constater, ce type de programme, bien qu'il prévoie des charges de cas allégées, n'en demeure pas moins très exigeant pour les intervenants. Soutien et encadrement sont donc absolument nécessaires pour permettre aux praticiens de maintenir une qualité d'intervention.

Il faut par ailleurs reconnaître que les programmes de sauvegarde de la famille ne sont pas la seule réponse à une situation de crise qui peut mener au placement d'un enfant, mais qu'ils sont plutôt des éléments d'un continuum de services offerts aux jeunes et à leurs familles. Ces programmes procèdent $d^{\prime}$ une philosophie qui permet d'aborder une crise familiale avec des objectifs de stabilité pour l'enfant et de maintien de l'entité familiale. Ils doivent cependant être vus en complémentarité avec d'autres services qui seront également toujours nécessaires pour certaines familles et certains enfants présentant des besoins particuliers. Ainsi, ce n'est pas parce que l'on veut éviter des placements que tous les placements doivent être évités. Dans certaines situations, le placement peut être une solution à court terme qui permet d'apporter une réponse immédiate à certains problèmes et de renforcer le milieu familial; dans d'autres cas, le placement sera la solution de choix pour assurer la sécurité de l'enfant.

Les évaluations des programmes de sauvegarde indiquent des taux de succès relatifs selon la nature des problèmes vécus dans le milieu familial. 
Ainsi les programmes n'ont pas toujours présenté la même efficacité à maintenir l'enfant dans sa famille lorsque les parents étaient responsables d'abus physiques sur leurs enfants ou lorsqu'ils s'agissait d'adolescents délinquants (Stroul, 1988). Les chercheurs américains ne se sont pas penchés spécifiquement sur la question de l'intervention en contexte d'autorité, mais certains résultats démontrent que les programmes sont moins efficaces lorsque les clients coopèrent moins avec les services (Fraser et al., 1991 ; Schwartz, AuClaire et Harris, 1991). Cela nous amène à émettre quelques réserves sur I'application de ces programmes dans les situations de prise en charge non volontaire.

Quoi qu'il en soit, les programmes de sauvegarde de la famille représentent un progrès intéressant dans le domaine de l'intervention auprès des familles à risque. Les recherches évaluatives menées jusqu'à ce jour sont suffisamment concluantes (même si elles devront être raffinées), pour que I'on tente d'implanter de tels programmes au Québec. L'expertise développée aux États-Unis devrait permettre d'en guider l'implantation à tous les niveaux ; puissions-nous, pour le bien-être des enfants et de leur famille, nous en inspirer.

\section{Références bibliographiques}

AuClaiRE, P. et I. SCHWARTZ (1986). An Evaluation of the Effectiveness of Intensive Home-Based Services as an Alternative to Placement for Adolescents and Their Families. Minneapolis : University of Minnesota, Center for Study of Youth Policy, H.H. Humphrey Institute of Public Affairs.

BARTH, R.P. (1990). « Theories Guiding Home-Based Intensive Family Preservation Services », dans J. K. WHITTAKER, J. KINNEY, E. M. TRACY et C. BOOTH (eds.), Reaching High Risk Families: Intensive Family Preservation in Human Services. New York : Aldine.

BeHAVIORAl SCIENCES INSTITUTE (1986). Homebuilders Cost Effectiveness with Various Client Populations. Federal Way, Washington : Behavioral Sciences Institute.

BeHAVIORAL SCIENCES INSTITUTE (1987). Homebuilders : An Overview. Federal Way, Washington : Behavioral Sciences Institute.

BEHAVIORAL SCIENCES INSTITUTE (1990). Homebuilders Evaluation Summary. Federal Way, Washington : Behavioral Sciences Institute.

BERRY, M. (1991). «The Assessment of Imminence of Risk of Placement : Lessons from a Family Preservation Program », dans Children and Youth Services Review, vol. $13: 239-256$.

BLYTHE, B. (1988). "Applying Practice Research Methods in Intensive Family Preservation Services », dans J. K. Whittaker et al. (eds.), Improving Practice Technology for Work with High Risk Families: Lessons from the "Homebuilders" Social Work Education Project. Seattle, WA : Center for Social Welfare Research. 
Blythe, B., TRACY, E. M., KOtOVSkY, A. et S. GWATKIN (1992). "Organizational Supports to Sustain Intensive Family Preservation Programs », Families in Society: The Journal of Contemporary Human Services, vol. 73, $\mathrm{n}^{\circ} 8: 463-470$.

BRIBITZER, M.P. et M.J. VERDIECK (1988). « Home-Based Family Centered Intervention : Evaluation of a Foster Care Prevention Program », Child Welfare, vol. 67, n 3 : 255-266.

BRUNER, C. (1988). The Decategorization of Child Welfare Services : A report of lowa's first year efforts. Washington, DC : Center for the Study of Social Policy.

BRUNER, C. (1989). Is Change from Above Possible? State-Level Strategies for Supporting Street-Level Services. Alexandria : Association for Public Policy Analysis and Management.

Bruner, C. et The Center for the Study of Social Policy (1988). Family Preservation Services in lowa : A Legislators Perspective on Key Issues. Washington, DC. : Center for the Study of Social Policy.

BRYCE, M. (1982). Home-Based Care: Directions for the 80s. Iowa City: The University of lowa, The National Resource Center on Family-Based Services.

BRYCE, M. et J. LlOYD (eds.) (1981). Treating Families in the Home: An Alternative to Placement. Springfield, IL : Charles C. Thomas, publ.

CHABOT, L. (1991). Symposium Garder ensemble les familles en difficulté : programmes prometteurs et réformes d'États. Traduction autorisée, réalisée par L. Chabot, Longueuil : CSSR.

COlE, E. et J. Duva (1990). Family Preservation : An Orientation for Administrators and Practitioners, Washington, DC : Child Welfare League of America.

DORE, M. (1991). «Context and Structure of Practice : Implications for Research on Intensive Family Preservation Programs », dans K. Wells et D. E. Biegel, Family Preservation Services, Newbury Park, CA : Sage Publications.

FANSHEL, D. et E. SHINN (1978). Children in Foster Care. A Longitudinal Investigation, New York : Columbia University.

FELDMAN, L.H. (1987). Using an Ecological Perspective to Identify Factors Influencing the Efficacy of Family-Based Intensive Services to Prevent Out of Home Placement. Trenton, NJ : New Jersey Division of Youth and Family Services.

FELDMAN, L.H. (1991). "Evaluating the Impact of Intensive Family Preservation Services in New Jersey ", dans K. Wells et D. E. Biegel (eds.), Family Preservation Services : Research and Evaluation. Newbury Park, CA : Sage Publications.

FRANKEL, H. (1988). "Family-Centered, Home-Based Services in Child Protection: A Review of the Research », Social Service Review, vol. 62 : 137-157.

Fraser, N.W., PeCORA, P.J. et D.A. HAAPALA (1991). Families in Crisis : The Impact of Intensive Family Preservation Services. Hawthorne, NY : Aldine de Gruyter.

FUQUA, R. (1988). lowa Family Preservation Project Evaluation Report. Ames, lowa : lowa State University.

Goldstein, J., Freud, A. et A. SOlNIT (1973). Before the Best Interests of the Child. New York : Free Press.

Gordon, D.A., TAButhnOt, J., Gustafson, K.E. et T. MCGreen (1988). " HomeBased Behavioral-Systems Family Therapy with Disadvantaged Juvenile Delinquants », American Journal of Family Therapy, vol. 16, no 3 : 243-255. 
HAAPALA, D. et J. KINNEY (1979). "Homebuilders Approach to the Training of In-Home Therapists », dans S. Maybanks et M. Bryce (eds.), Home-Based Services for Children and Families, Springfield, IL : Charles C. Thomas, publ.

HAAPALA, D. et M. FrASER (1987). Client and Family Therapist Perspectives on HomeBased Family Therapy: Differences By Treatment Outcomes. Federal Way, WA : Behavioral Sciences Institute.

HAAPALA, D., KINNEY, J. et K. MCDADE (1988). Refering Families to Intensive HomeBased Family Preservation Services : A Guide Book. Federal Way, WA : Behavioral Sciences Institute.

HARTLEY, R., SHOWELl, B. et J. White (1989). "Outcomes of Oregon's Family Treatment Programs: A Descriptive Study of 1752 Families», Background Papers. Intensive Family Preservation Services Research Conference, Cleveland, Ohio : Case Western University.

HAWKINS, J.W. et R.F. CATALANO (1990). "Intensive Family Preservation Services : Broadening the Vision for Prevention », dans J.K. Whittaker, J. Kinney, E. Tracy, C. Booth (eds.), Reaching High Risk Families : Intensive Family Preservation in Human Services. New York: Aldine de Gruyter.

HINCKLEY, E. (1984). "Homebuilders: The Maine Experience », Children Today, sept.-oct. $1984: 14-17$.

JONES, M.A. (1985). A Second Chance for Families, Five Years Later : Follow-Up of a Program to Prevent Foster Care. New York : Child Welfare League of America.

KINNEY, J. et D. HAAPALA (1988). "Avoiding Out-of-Home Placement of High-Risk Status Offenders through the Use of Intensive Home-Based Services », Criminal Justice and Behavior, vol. $15: 334-348$.

KinNeY, J., HAAPALA, D., BOOTH, C. et S. LeAvitT (1990). "The Homebuilders Model », dans J.K. Whittaker, J. Kinney, E. Tracy, C. Booth (eds.), Reaching High Risk Families : Intensive Family Preservation in Human Services. New York : Aldine de Gruyter : 31-64.

KINNEY, J., MADSEN, B., FleminG, T. et D. HAAPAla (1977). " Homebuilders : Keeping Families Together », Journal of Consulting and Clinical Psychology, vol. 45 : 667-673.

KNITZER, J. et E. COLE (1989). Family Preservation Services: The Policy Challenge to State Child Welfare and Mental Health Systems, Report from Changing Services for Children, New York : Bank Street College of Education.

KOSHEL, J. et J. KINNEY (1986). Family Preservation : The Homebuilders' Program in the State of Washington. Washington, DC : National Governors' Association.

LANDSMAN, M.J. (1985). Evaluation of Fourteen Child Placement Prevention Projects in Wisconsin. 1983-1985. lowa City: University of lowa, The National Resource Center on Family-Based Services.

LEEDS, S. (1984). Evaluation of Nebraska's Intensive Services Project, lowa City : University of lowa, The National Resource Center on Family-Based Services.

MAGURA, S. (1981). "Are Services to Prevent Foster Care Effective? », Children and Youth Services Review, vol. 3 : 193-212.

MAGURA, S. et B.S. MOSES (1986). Outcome Measures for Child Welfare Services : Theory and Applications. Washington, DC : Child Welfare League of America. 
MAGURA, S., MOSES, B.S. et M.A. JONES (1987). Assessing Risk and Measuring Change in Families : The Family Risk Scale. Washington, DC : Child Welfare League of America.

MALuCCIO, A. (1988). "Family Preservation Services and the Social Work Practice Sequence », dans J. Whittaker et al. (eds.), Improving Practice Technology for Work with High Risk Families : Lessons from the "Homebuilders " Social Work Education Project, Seattle, WA : Center for Social Welfare Research.

MAluCCiO, A., Fein, E. et K.A. OlmSteAd (1988). Permanency Planning for Children : Concepts and Methods. New York : Tavistock Publ.

MARYLAND DEPARTMENT OF HuMAN ReSOURCES (1987). Intensive Family Services : A Family Preservation Service Delivery Model. Manuel préparé par les Services aux familles avec enfants, Maryland: Department of Human Resources.

NATIONAL COMmisSiOn OF CHILDREN IN NeED OF PARENTS (1978). Who Knows? Who Cares? Forgotten Children in Foster Care. Washington, DC.

National ResourCe Center on Family-BASed Services (1989). An Analysis of Factors Contributing to Failure in Family-Based Child Welfare Services in Eleven Family-Based Agency : Final Report. lowa City.

NeLSON, K.E. (1988). Family as Patient of Therapeutic Ally: The Family-Based Services Perspective, Presented at the National Symposium on Child Victimization, Anaheim, CA.

NELSON, K.E. (1990). " How Do We Know that Family-Based Services Are Effective? ", Prevention Report. Oakdale, IA : National Resource Center on Family-Based Services, $1: 1-3$.

NeLSON, K.E. (1990). "Recognizing and Realizing the Potential of 'Family Preservation' », dans J.K. Whittaker et al., Reaching High Risk Families : Intensive Family Preservation in Human Services. New York : Aldine de Gruyter.

NeLSON, K.E. (1990). «Program Environment and Organization », dans Y.-Y. T. Yuan et M. Rivest, Preserving Families : Evaluation Resources for Practitioners and Policymakers. Newbury Park, CA : Sage Publications.

NELSON, K.E. (1991a). "Populations and Outcomes in Five Family Preservation Programs », dans K. Wells et D.E. Biegel (eds.), Family Preservation Services : Research and Evaluation. Newbury Park, CA : Sage Publications.

NeLSON, K.E. (1991b). Assessing the Risk of Placement in Family Preservation Services. National Resource Center on Family-Based Services, University of lowa.

NelSON, K.E., EMLEN, A., LANDSMAN, M.J. et J. HutCHINSON (1988). Factors Contributing to Success and Failure in Family-Based Child Welfare Services. lowa City, IA : National Resource Center on Family-Based Services.

NelsON, K.E., LANDSMAN, M.J. et W. DeutelBaum (1990). « Three Models of FamilyCentered Placement Prevention Services », Child Welfare, vol. LXIX, nº $1: 3-21$.

OOMS, T. (1990). The Crisis in Foster Care: New Directions for the 1990s. Meeting Highlights and Background Briefing Report, Family Impact Seminar, AAMFT Research and Education Foundation, Washington, DC.

OOMS, T., BeCK, D. et L. HeREndeEn (1990). Family Impact Seminar, AAMFT Research and Education Foundation, Washington, DC.

PeCORA, P. (1988). « Designing and Managing Family Preservation Services : Implications for Human Services Administration Curricula », dans J. Whittaker et al. (eds.), Improving Practice Technology for Work with High Risk Families : Lessons from the "Homebuilders "Social Work Education Project. Seattle, WA : Center for Social Welfare Research. 
Pecora, P., Delewski, C., BoOth, C., HaApala, D. et J. Kinney (1985). " HomeBased Family-Centered Services: The Impact of Training on Worker Attitudes », Child Welfare, vol. 64, no $5: 529-540$.

Pecora, P., Fraser, M., HaApala, D. et J. Bartlome (1987) «Defining Family Preservation Services: Three Intensive Home-Based Treatment Programs ». South Lake City : University of Utah Social Research Institute, cité dans M. Berry (1991).

PeCorA, P., KInNeY, J., MitChell, L. et G. TOlley (1990). "Selecting an Agency Auspice for Family Preservation Services », Social Service Review, vol. 64, $\mathrm{n}^{\circ} 2: 288-307$.

PeCORA, P., Fraser, M. et D. HAAPAla (1991). «Intensive Home-Based Treatment : Client Outcomes and Issues for Program Design », dans K. Wells et D. E. Biegel (eds.), Family Preservation Services : Research and Evaluation. Newbury Park, CA : Sage Publications.

ReID, W.J., KAGAN, R.M. et S.B. SCHLOSBERG (1988). "Prevention of Placement: Critical Factors in Program Success », Child Welfare, vol. 67, $\mathrm{n}^{\circ} 1: 25-36$.

Rossı, P. (1991). Evaluating Family Preservation Projects : A Report to the Edna McConnell Clark Foundation. Amherst : The University of Massachusetts.

Rossı, P. (1992). "Assessing Family Preservation Programs », Children and Services Review, vol. 14 : 77-97.

SCHUERMAN, J.R., RZEPNICKI, T. et J. LITTELL (1991). "From Chicago to Little Egypt : Lessons from an Evaluation of a Large Scale Child Welfare Family Preservation Program », dans K. Wells et D.E. Biegel (eds.), Family Preservation Services : Research and Evaluation, Newbury Park, CA : Sage Publications.

SChWARTZ, I., AuClaire, P. ET L.J. HARRIS (1991). « Family Preservation Services as an Alternative to the Out-of-Home Placement of Adolescents: The Hennepin County Experience », dans K. Wells et D.E. Biegel (eds.), Family Preservation Services : Research and Evaluation. Newbury Park, CA : Sage Publications.

SPAID, W.M. et M. FrASER (1991). "The Correlates of Success/Failure in Brief and Intensive Family Treatment : Implications for Family Preservation Services », Children and Youth Services Review, vol. 13 : 77-99.

Stroul, B.A. (1988). " Home-based services », Series on Community-Based Services for Children and Adolescents Who Are Severely Emotionally Disturbed (vol. 1). Washington, DC : CASSP Technical Assistance Center, Georgetown University Child Development Center.

TAVANTZIZ, T.N., TAVANTZIZ, M., BROWN, L.G. et M. ROHRBAUGH (1985). " HomeBased Structural Family Therapy for Delinquents at Risk of Placement », dans M.P. Murkin et L. Koman (eds.), Handbook of Adolescents and Family Therapy. New York : Gardner.

THIEMAN, A.A., FUQUA, R. et K. LINNAN (1990). lowa Family Preservation Three Year Pilot Project : Final Evaluation Report. Ames, IA : Child Welfare Research and Training Project, lowa State University.

Thieman, A.A. et P.W. DAll (1992). « Family Preservation Services : Problems of Measurement and Assessment of Risk», Family Relations, vol. 41 : 186-191.

TRACY, E. (1991). "Defining the Target Population for Family Preservation Services », dans K. Wells et D.E. Biegel (eds.), Family Preservation Services : Research and Evaluation. Newbury Park, CA : Sage Publications. 
TYLER, M. (1991). Intensive Family Services Research Project : Preliminary Report, lowa City, IA : National Resource Center on Family-Based Services.

WAld, M. (1988). "Family Preservation : Are We Moving Too Fast ? ", Public Welfare, vol. 46, n $3: 33-38$.

WeLLS, K. et D.E. BIEGEL (1989). Intensive Family Preservation Services : A Research Agenda for the 1990s. Final Report, Intensive Family Preservation Services Conference, Ohio.

WELLS, K. et D.E. BIEGEL (1991). Family Preservation Services : Research and Evaluation. Newbury Park, CA : Sage Publications.

WHITTAKER, J.K., KINNEY, J., TRACY, E.M. et C. BOOTH (1988). Improving Practice Technology for Work with High Risk Families: Lessons from the "Homebuilders" Social Work Education Project. Seattle, WA : Center for Social Welfare Research.

WHITTAKER, J.K., KINNEY, J., TRACY, E.M. et C. BOOTH (1990). Reaching High Risk Families : Intensive Family Preservation in Human Services. New York : Aldine de Gruyter.

WHITTAKER, J.K. (1991). «The Leadership Challenge in Family-Based Services : Policy, Practice and Research », Families in Society: The Journal of Contemporary Human Services, vol. 72, $\mathrm{n}^{\circ} 5: 298$.

YUAN, Y.-Y.T. (1990). "Cost analysis », dans Y.-Y.T. Yuan et M. Rivest (eds.), Preserving Families : Evaluation Resource for Practitioners and Policymakers. Newbury Park, CA : Sage Publications.

YUAN, Y.-Y.T. et M. RIVEST (eds.) (1990). Preserving Families : Evaluation Resources for Practioners and Policymakers. Newbury Park, CA : Sage Publications.

YUAN, Y.-Y.T. et D.L. STRUCKMAN-JOHNSON (1991). "Placement Outcomes for Neglected Children with Prior Placements in Family Preservation Programs », dans K. Wells et D.E. Biegel (eds.), Family Preservation Services : Research and Evaluation. Newbury Park, CA : Sage Publications. 